\title{
EVA Design, Verification, and On-Orbit
Operations Support Using Worksite Analysis \\ EVA Design, Verification, and On-Orbit
Operations Support Using Worksite Analysis
}

Thomas J. Hagale

The Boeing Company Huntsville, Alabama

Larry R. Price

The Boeing Company Huntington Beach, California 
SAE routinely stocks printed papers for a period of three years following date of publication. Direct your orders to SAE Customer Sales and Satisfaction Department.

Quantity reprint rates can be obtained from the Customer Sales and Satisfaction Department.

To request permission to reprint a technical paper or permission to use copyrighted SAE publications in other works, contact the SAE Publications Group.

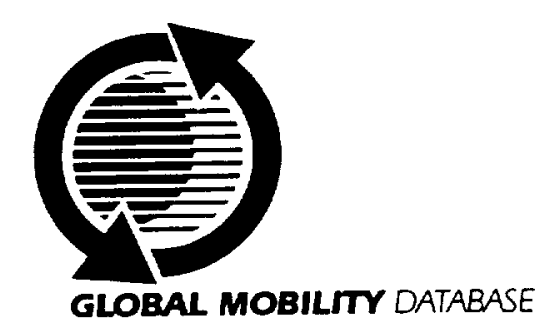

All SAE papers, standards, and selected books are abstracled and indexed in the Global Mobility Database

\section{ISSN 0148-7191}

Positions and opinions advanced in this paper are those of the author(s) and not necessarily those of SAE. The author is solely responsible for the content of the paper. A process is available by which discussions will be printed with the paper if it is published in SAE Transactions. For permission to publish this paper in full or in part, contact the SAE Publications Group.

Persons wishing to submit papers to be considered for presentation or publication through SAE should send the manuscript or a 300 word abstract of a proposed manuscript to: Secretary, Engineering Meetings Board, SAE.

\section{Printed in USA}




\title{
EVA Design, Verification and On-Orbit Operations Support Using Worksite Analysis
}

\author{
Thomas J. Hagale \\ The Boeing Company, Huntsville, AL \\ Larry R. Price \\ The Boeing Company, Huntington Beach, CA
}

\begin{abstract}
The International Space Station (ISS) design is a very large and complex orbiting structure with thousands of Extravehicular Activity (EVA) worksites. These worksites are used to assemble and maintain the ISS. The challenge facing EVA designers was how to design, verify, and operationally support such a large number of worksites within cost and schedule. This has been solved through the practical use of computer aided design (CAD) graphical techniques that have been developed and used with a high degree of success over the past decade. The EVA design process allows analysts to work concurrently with hardware designers so that EVA equipment can be incorporated and structures configured to allow for EVA access and manipulation. Compliance with EVA requirements is strictly enforced during the design process. These techniques and procedures, coupled with neutral buoyancy underwater testing, have proven most valuable in the development, verification, and on-orbit support of planned or contingency EVA worksites.
\end{abstract}

\section{INTRODUCTION}

With so many worksites to assess, an accurate and reliable analysis technique had to be developed. To fully implement the process, engineers need a good working knowledge of CAD. The techniques have been tailored specifically for the $C A D$ system being used for the worksite assessment. Three-dimensional solid CAD models are assembled to represent the flight hardware being investigated. 3-D models of crewmembers are imported to assess the worksites. EVA hardware is added to aid the crewmember. The analytical process is graphical and iterative, allowing the analyst to try different crewmember orientations until an optimum crew position is determined and EVA aids are positioned appropriately to support the tasks at the worksite.

\begin{abstract}
A key part of the process involves correctly identifying worksites needed for the assembly and maintenance of space hardware by EVA. Specific knowledge and experience with EVA helps with this identification. Since space-suited crewmembers are required for these assembly and maintenance tasks, accurate modeling of work envelopes for a range of astronauts is required. This has been accomplished through the careful integration of EVA requirements from Space Shuttle System Payload Accommodations (NSTS 07700), International Space Station Flight Crew Integration Standard (SSP 50005) and Extravehicular Activity (EVA) Standard Interface Control Document (SSP 30256) into the 3-D crew models. Most activities also require the use of tools, which in turn requires that the crewmembers' feet be restrained to operate those tools. Use of an Articulating Portable Foot Restraint, or APFR, accomplishes this requirement. The APFR, EVA tools, work envelopes, and $95^{\text {th }}$ percentile volumetric crewmember have been modeled and represented in the analysis.
\end{abstract}

\section{EVA WORKSITE ANALYSIS USING CAD}

The challenge created by so many worksites, coupled with the cost and schedule constraints of the ISS program, led engineers to develop a low cost, reliable method of designing and verifying EVA worksites. Traditionally, NASA and its contractors would use neutral buoyancy underwater development testing as the primary means of designing EVA worksite interfaces. This required placing space-suited crewmembers and fullscale mockup hardware in an underwater, neutrally buoyant environment to perform first-hand evaluations of the crew-to-hardware interfaces. Often, however, this testing could not be performed until the flight design had matured enough to build accurate mockups. At that point in design maturity, changes to the flight design would become very expensive. 
This design maturity dilemma coupled with cost and schedule constraints and the large number of ISS worksites did not allow for the extensive use of neutral buoyancy development testing. Therefore, a CAD EVA worksite analysis system was developed and used to design and verify EVA worksites as an adjunct to underwater neutral buoyancy, KC-135 zero-g simulator and air bearing floor testing. This often allowed issues to be analyzed and resolved more quickly than would have been possible using neutral buoyancy testing, resulting in less risk of design changes at a late point in the program. It also allowed neutral buoyancy testing, $\mathrm{KC}-135$ and air bearing floor testing to focus on critical evaluations that could not be fully addressed by CAD EVA worksite analysis. The result was optimum utilization of testing time for the difficult cases. A means of recording and publishing the results was also created to document the analyses and make them readily available to internal and external customers.

This methodology also proved to be a useful tool in quickly assessing on-orbit contingency operations or perturbations that arose during early ISS missions.

METHOD - The EVA designer begins with CAD models of the flight hardware currently in development. EVA tasks are identified and a rough outline of the task scenario is created. Specially designed models of $95^{\text {th }}$ percentile space suited crewmembers in APFRs are imported into the hardware assembly model to assess reach and access to the worksite. This model of the crewmember contains envelopes that depict one- and two-handed work volumes (see Figures 1 and 2) for astronauts in the range of $5^{\text {th }}$ to $95^{\text {th }}$ percentile male crewmember. The work envelopes are oriented in a specific geometric relationship with the APFR footplate as defined in NSTS 07700 .

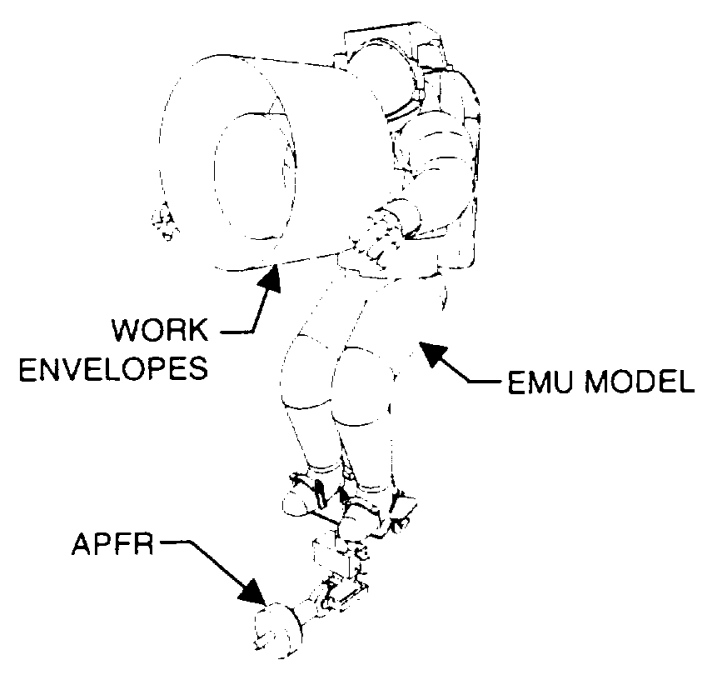

Figure 1. EMU in APFR with Work Envelopes

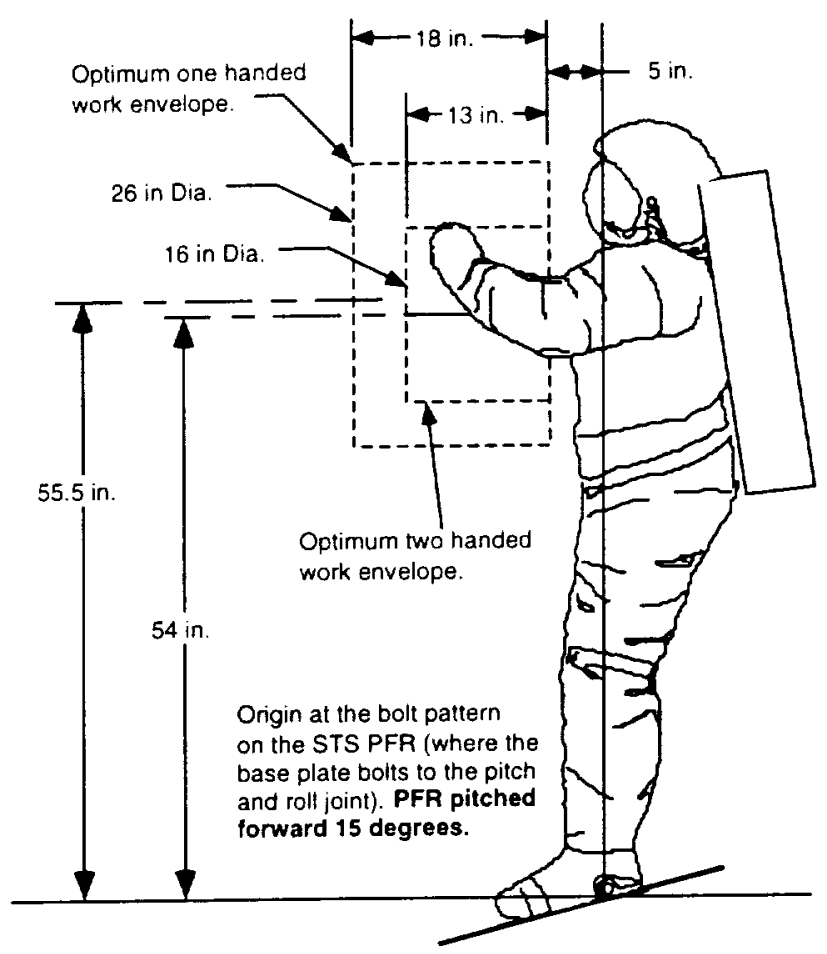

Figure 2. Crewmember Optimum Work Envelope

By using a specially designed $C A D$ routine, the analyst manipulates the crewmember model by articulating the foot restraint, or APFR, to any one of many different configurations to optimize reach and access to the worksite. Precisely 33,264 unique combinations of roll, pitch, and yaw exist for the APFR. This optimization involves continuous dialogue with the hardware designers to insure a good concurrent design process.

The investigation reveals where APFR worksite interface (WIF) sockets should be installed on structure and where handrails should be placed to support translation, ingress and egress of the APFR, APFR installation, and crewmember stabilization at the worksite.

Validity of Model and Analysis Technique - The graphical analytical technique described in this paper uses 3D CAD models of space flight hardware, the NASA Extravehicular Mobility Unit (EMU), APFR, and various EVA aids and tools. NASA validated this technique during the ISS Design Analysis Cycle 2 (DAC-2) in 1995 (reference 4). The report concluded that the technique to date had a performance accuracy of $90 \%$ when used within the constraints specified in the DAC-2 report. This was based on a comparison of the analytical predictions of the crew to do a task and actual neutral buoyancy water test results for 6 worksites and approximately 30 crew positions. Comparison of the CAD drawing in Figure 3 and the photo in Figure 4 is an example of good correlation between analysis prediction and neutral buoyancy test. 


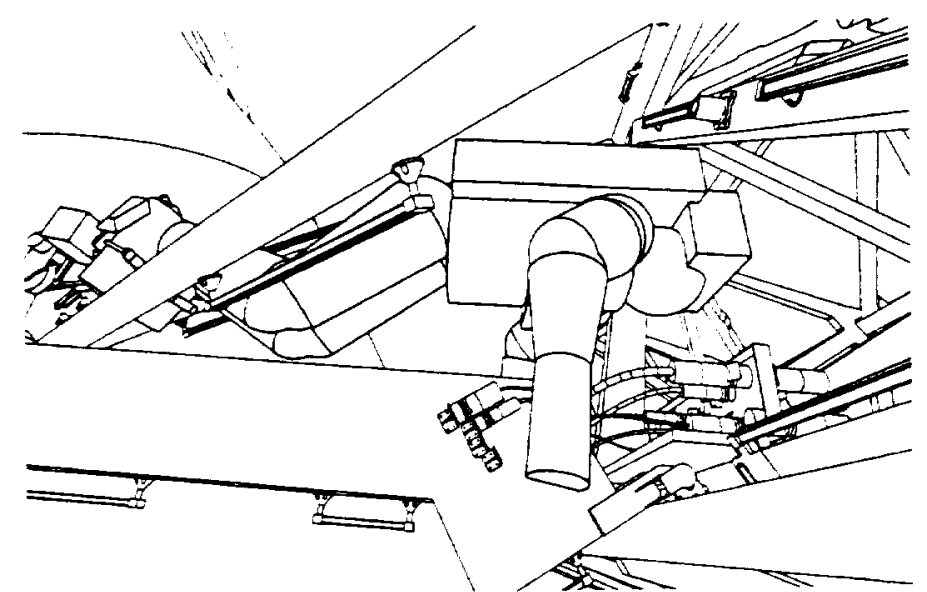

Figure 3. Habtray Installation - CAD EVA Analysis

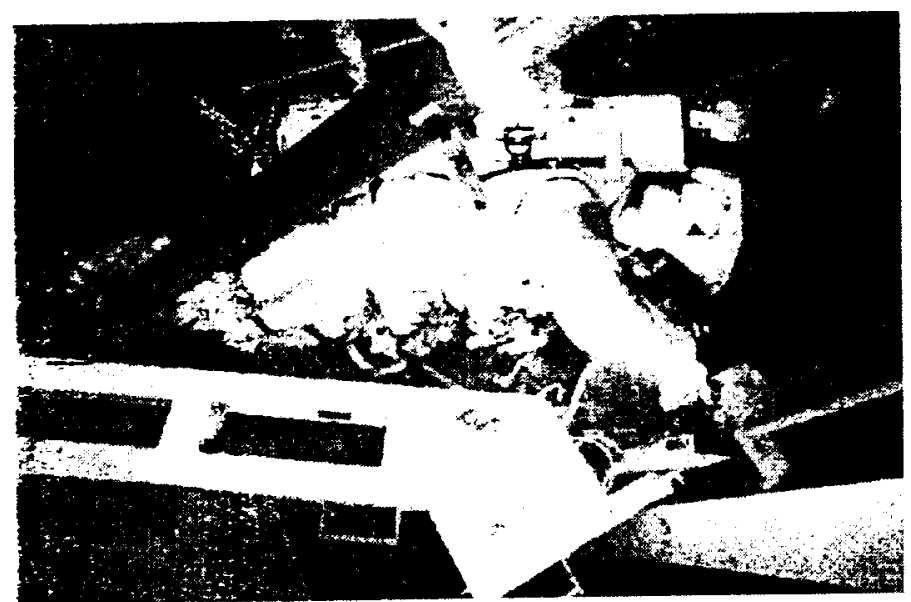

Figure 4. Habtray Installation - Underwater Testing

Guidelines for use of CAD EVA worksite analysis were established and investigated in the DAC-2 report. Requirements that were found suitable to be addressed by CAD EVA analysis included: dedicated worksite operations within the optimal work envelope, reach and access, lighting geometry, handrail and worksite interface placement and quantity, glove clearance, working volume and translation path diameter. Crewmember field of view was initially established as acceptable, but the results of testing indicated that sufficient visibility could not be completely predicted by CAD analysis and should be used with caution.

Requirements found unsuitable to be addressed by CAD EVA analysis included: dexterity and handling, mass handling, forces and torque in multiple axes with a volume greater than 5 cubic feet and/or a mass greater than 50 lbs., alignment tolerances, use of non-standard controls and indicators, mental and physical fatigue levels, operational functionality, timeline determination and manipulation of flexible hardware (cables, umbilicals, or tethers)

A later NASA report (reference 5) shows data comparing $A P F R$ settings from $C A D$ analysis with actual settings used in 5 different neutral buoyancy tests for 90 tasks with 447 separate evaluations. Results were consistent with the DAC-2 validation report.

DESIGN AND VERIFICATION TOOL - Each worksite analysis is started by categorizing the tasks as either assembly or maintenance, determining if the tasks should be handled by EVA, and identifying the worksite location and hardware configuration at the time of the proposed EVA. The analyst contacts the designers involved with the hardware being assembled, deployed, stowed, or replaced. Models and pertinent information about the tasks are acquired from the designers and an assembly is created electronically in the CAD system. The on-orbit hardware configuration is important for the validity of the EVA worksite analysis. Therefore, the actual flight during which the EVA will be performed in the assembly sequence must be known prior to conducting the analysis. Assumptions are made and documented (including the hardware configuration) to provide a basis for the investigation.

A decision must be made concerning how the worksite tasks will be performed. The choice is whether the crewmember will be restrained (on the robotic arm or in an APFR) or free-float. This may have been predetermined for the worksite or open for assessment. The type of tasks being performed will ultimately determine the EVA method.

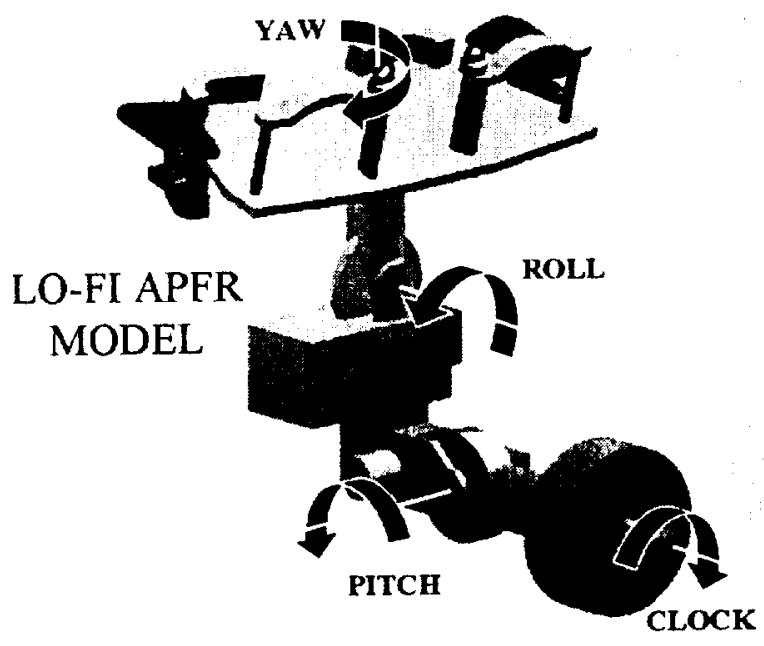

Figure 5. Articulating Portable Foot Restraint

Next, the analyst must import one or more EV (Extravehicular) crewmembers into the assembly. The EV crewmember consists of an Extravehicular Mobility Unit (EMU) and an APFR. A program for importing and manipulating the EV crewmember is used as an aid to the analyst. Once the EV crewmember is imported (and automatically named) it may be manipulated using the same program. The routine allows the analyst to rotate the APFR in the clock, pitch, roll, and yaw axes (see Figure 5). Since the EMU is restrained on the footplate of the APFR, it moves as the APFR is articulated. The program allows the analyst to quickly manipulate the 
EMU into several positions until access to the worksite is optimized. Multiple iterations may be performed to adjust the APFR and/or socket locations on the structure to achieve the desired results. In the past this was an intuitive trial and error process which often took a great deal of time. Recently a sub-program has been developed which automatically and quickly checks all possible APFR positions and identifies the best positions available.

Finally, mobility aids such as handholds and handrails. and access and clearance envelopes are assessed. If the APFR is based in a socket on the structure, a translation path to the worksite must be identified. Also. the work envelope must encompass the hardware or tool that the EV crewmember will grasp to perform the task. This insures that the full range of astronauts can comfortably manipulate the hardware or tool at the worksite.

NEUTRAL BUOYANCY TESTING - Neutral Buoyancy Laboratory (NBL) testing remains a preferred method for astronauts to physically assess the viability of worksites and is used as cost and schedule allow. The CAD worksite analysis serves double duty in this case by providing a basis, or starting point, for NBL testing complete with handrail and WIF socket locations, APFR settings and suggested task procedures. Types and combinations of tools are also investigated and recommended.

As the hardware design matures and NBL testing draws near, the EVA analyst prepares a preliminary worksite analysis. When complete, this analysis flows directly into the NBL test plan and describes tasks, crew positions. APFR settings, and EVA tools and aids. Full color prints of the crew and hardware configurations may be submitted for use in the detailed test procedures created by the NBL test team.

Depending on the fidelity of the analysis and the accuracy of the NBL mockups, usually few to no APFR settings adjustments are necessary to accommodate each crewmembers' reach and access at the worksites analyzed. Figures 6 and 7 demonstrate the similarities between analysis modeling and NBL reality.

A recent NBL test of the removal and repiacement of an ammonia tank assembly from the unpressurized carrier used to transport it to orbit resulted in a $100 \%$ acceptance by the crew of the CAD analyzed APFR settings.

The use of CAD EVA worksite analysis has proven to be a most valuable asset in optimizing the time the astronauts spend in the water and in providing more accurate EVA hardware installations.

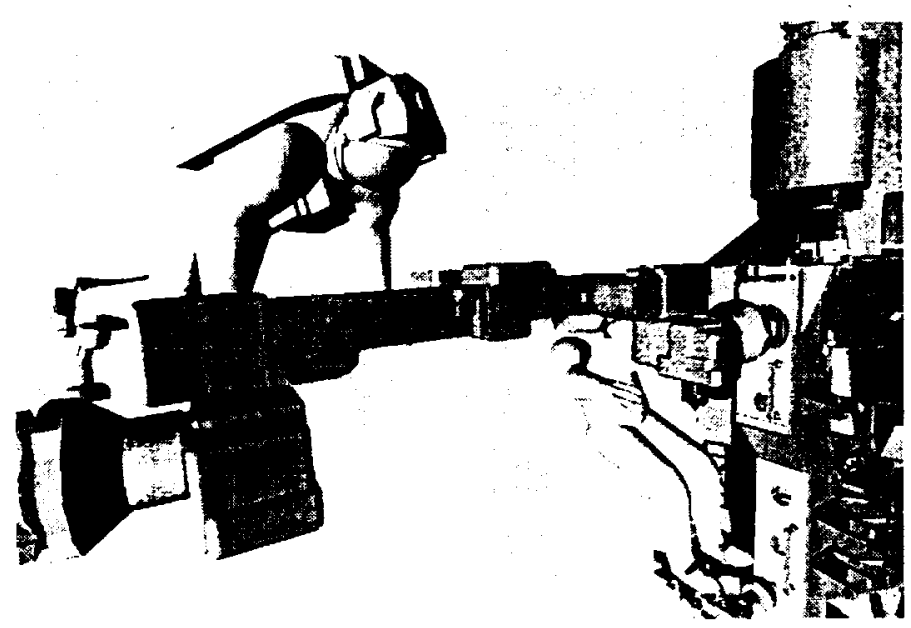

Figure 6. SPDM Arm Temp Stow - CAD Analysis

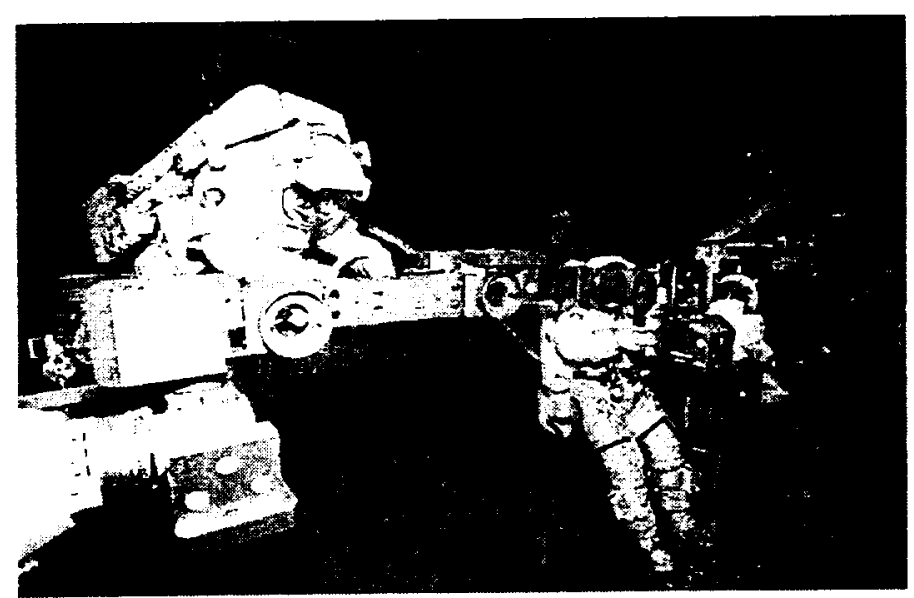

Figure 7. SPDM Arm Temp Stow - NBL Testing

ON-ORBIT OPERATIONS SUPPORT - This graphical worksite analysis tool is also a great help while supporting the conduct of planned and contingency EVA operations on-orbit. It allows rapid understanding and visualization of planned operations using previously completed analyses or it allows quick real time investigation of unexpected problems that may arise. Many of the completed analyses have already been used to provide significant information to engineering personnel in support of the following early ISS missions.

- Flight 2A (STS 88): Launch of the U.S. element Unity and assembly of Unity to the Russian element Zarya.

- Flight 2A.1 (STS 96): Shuttle launch to ISS to allow the Astronauts to accomplish repairs.

- Flight 2A.2A (STS 101): Shuttle launch to ISS to allow the astronauts to accomplish further repairs and prepare UNITY/ZARYA for the assembly of the next ISS element. 


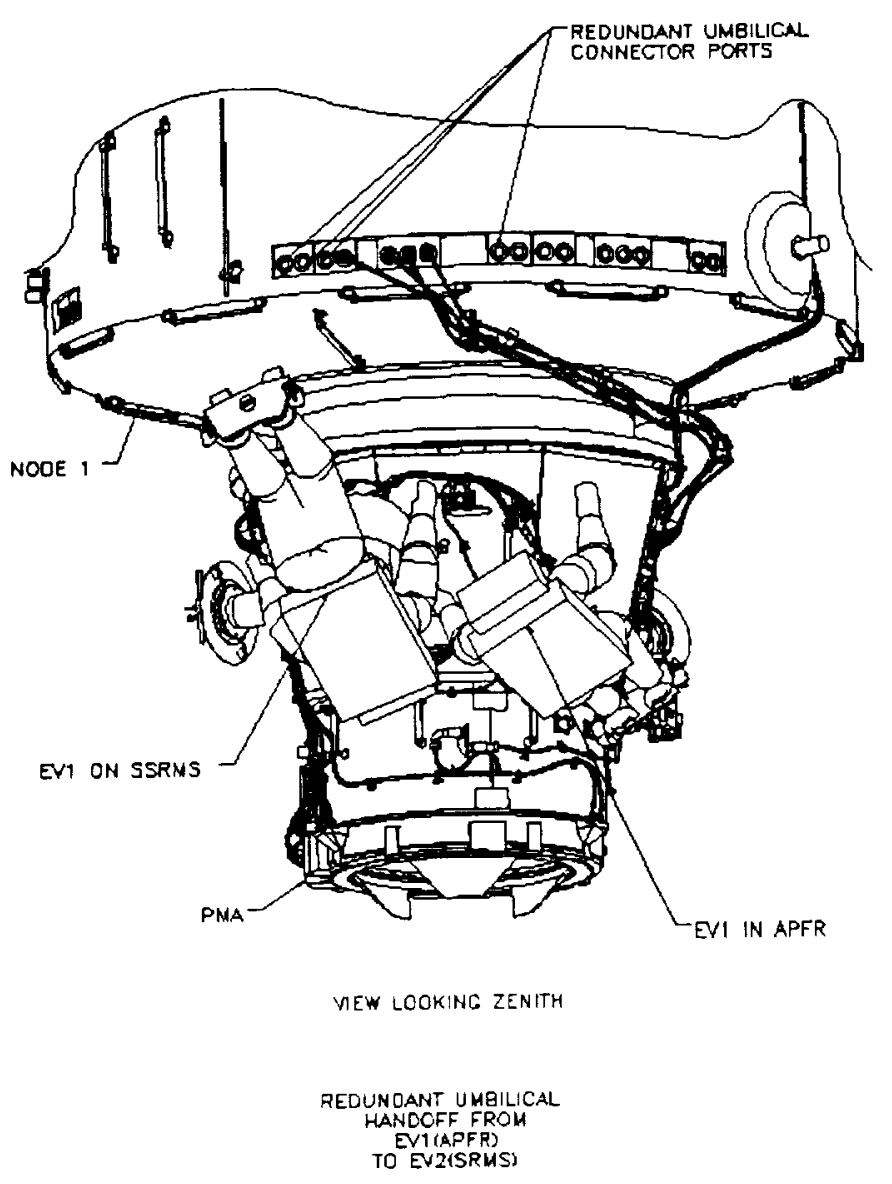

Figure 8. EVA Tasks on Flight 2A

For example, 24 (of 120 total) completed analyses were actively run on ground based computers at NASA JSC and Boeing during the various EVA operations on Flight 2A. These analyses were used to verify and sometimes define the following types of EVA tasks:

- Access equipment and stowage areas

- Locate EVA aids

- Accomplish equipment maintenance

- Install/remove electrical jumpers, equipment and attachment devices

In the future it is expected that, with a worksite analysis file actively running on the ground-based CAD system, alterations and instructions from the on-orbit crew can be fed directly into the analysis and alternate solutions suggested. This tool could also be used to help prepare the crew for the worksite tasks and configurations prior to leaving the airlock.

\section{CONCLUSION}

By making use of the CAD EVA analysis process, design time and verification can be greatly reduced and simplified. Also, involving the hardware designers early in the design process can minimize subsequent costly changes to the hardware, thereby efficiently utilizing the concurrent design philosophy.

On-orbit support is greatly enhanced by the use of CAD analysis to recreate in-flight situations on Earth and quickly research alternative solutions in real-time. Additionally, graphical representations of the assembly or maintenance process can be up-linked directly to the onorbit crew for review prior to or during an EVA.

Ten years of developing and using CAD EVA worksite analysis, coupled with recent on-orbit experience, has shown that this methodology is a cost effective and reliable process that accurately predicts and depicts onorbit operations. It has also been proven to correlate well with the results of NBL testing.

\section{ACKNOWLEDGMENTS}

The authors would like to thank Amy Heartsill, Tammy Hinkle and Mike Fruhwirth for their help in reviewing, editing and contributing to this paper. We also wish to recognize NASA and their space programs through which the techniques described in this paper were made possible.

\section{REFERENCES}

1. Thomas J. Hagale, "International Space Station Computer Aided Extravehicular Activity Worksite Analysis," 96-1-07. Proceedings of the 20th International Symposium on Space Technology and Science. Gifu, Japan, 1996.

2. Space Shuttle System Payload Accommodations, NSTS 07700 , Volume XIV, Appendix 7, Revision J., 1988.

3. Larry R. Price, Michael A. Fruhwirth and Jeffrey G. Knutson: "Computer Aided Design and Graphics Techniques for EVA Analysis." ISSN0148-7191. 24 ICES and $5^{\text {th }}$ ESSECS, 1994.

4. "Validation Analysis of PG-1 EMU and EVA Tools 3D Models," ISS Design Analysis Cycle 2, TDS 3.1.14-3, August 1995.

5. Susan Hatcliff, "Unigraphics Analysis Modeling and Weightless Environment Training Facility Comparison Data". NASA JSC, CTSD-SS-885, February 1996.

6. "International Space Station Flight Crew Integration Standard", SSP 50005, Revision B.

7. "EVA Standard Interface Control Drawing", SSP 30256 Revision G, June 1999. 


\section{CONTACT}

Thomas J. Hagale has a Bachelor of Science degree in Aerospace Engineering from the University of Missouri Rolla, USA, 1979. Mr. Hagale has worked in the aerospace industry for 21 years with the last 10 specializing in EVA design, analysis and integration for the International Space Station. He currently works for the Boeing Company in Huntsville, Alabama.

E-mail: thomas.j.hagale@boeing.com

Phone: 256-461-3687
DEFINITIONS, ACRONYMS, ABBREVIATIONS

APFR Articulating Portable Foot Restraint

CAD Computer Aided Design

DAC Design Analysis Cycle

EMU Extravehicular Mobility Unit

EV Extravehicular

EVA Extravehicular Activity

ISS International Space Station

JSC Johnson Space Center

NASA National Aeronautics and Space Administration

NBL Neutral Buoyancy Laboratory

NSTS National Space Transportation System

SPDM Special Purpose Dexterous Manipulator

SSP Space Station Program

STS Space Transportation System

WIF Worksite Interface 